\title{
Correction to: Cognitive assessment in multiple sclerosis-an Italian consensus
}

\author{
Maria Pia Amato ${ }^{1,2}$. Vincenzo Brescia Morra ${ }^{3}$ - Monica Falautano ${ }^{4}$ - Angelo Ghezzi ${ }^{5}$ - Benedetta Goretti ${ }^{1}$. \\ Francesco Patti ${ }^{6}$ - Alice Riccardi ${ }^{7}$. Flavia Mattioli ${ }^{8}$
}

Published online: 21 March 2019

(C) Fondazione Società Italiana di Neurologia 2019

\section{Correction to: Neurological Sciences (2018) 39:1317-1324 https://doi.org/10.1007/s10072-018-3427-x}

In the original article, Maria Pia Amato's second affiliation was not included. The second affiliation is IRCCS Fondazione Don Carlo Gnocchi, Florence, Italy. The correct affiliation is presented here.

Publisher's note Springer Nature remains neutral with regard to jurisdictional claims in published maps and institutional affiliations.

The online version of the original article can be found at https://oi.org/ 10.1007/s10072-018-3427-x

Maria Pia Amato

mariapia.amato@unifi.it

1 Department of NEUROFARBA, University of Florence,

Florence, Italy

2 IRCCS Fondazione Don Carlo Gnocchi, Florence, Italy

3 Department NSRO, University of Naples, Naples, Italy

4 Department of Neurology, INSPE, Vita-Salute University and IRCCS-San Raffaele Hospital, Milan, Italy

5 Centro Studi Sclerosi Multipla, Gallarate Hospital Vita-Salute University, Gallarate, Italy

6 Department of Neurosciences G.F. Ingrassia, University of Catania, Catania, Italy

7 Multiple Sclerosis Centre, Department of Neurosciences-DNS, Padova, Italy

8 Neuropsychology Unit, Spedali Civili of Brescia, Brescia, Italy 